\title{
DESEMPENHO EM CONFINAMENTO DE MACHOS BOVINOS INTEIROS CANCHIM, ABERDEEN ANGUS E CRUZAMENTOS RECÍPROCOS
}

\author{
FEEDLOT PERFORMANCE OF CANCHIM, ABERDEEN \\ ANGUS AND RECIPROCAL CROSSBRED MALES
}

\author{
Daniel Perotto $^{1}$ José Luiz Moletta $^{2}$ Carlos Lesskiu $^{3}$
}

\section{RESUMO}

Foram analisados o consumo diário de matéria seca (MS) por $100 \mathrm{~kg}$ de peso vivo (CMS), a conversão alimentar (CA) e o ganho de peso médio diário (GMD) de 118 machos bovinos inteiros Canchim (Cn), Aberdeen Angus (Ab) e cruzamentos reciprocos (CnAb (F $, 3 / 4 C n+1 / 4 A b, \quad 5 / 8 C n+3 / 8 A b \quad e$ $11 / 16 C n+5 / 16 A b) \quad e \quad A b C n \quad\left(F_{l}, \quad 5 / 8 A b+3 / 8 C n \quad e\right.$ $11 / 16 A b+5 / 16 C n))$. Esses animais foram alimentados em baias individuais por 84 a 95 dias com silagem de milho à vontade mais concentrado $(17,8 \%$ de $P B$ e $79 \%$ de NDT) fornecido à base de $1 \%$ do peso vivo do animal por dia. As características foram analisadas por um modelo que incluiu os efeitos fixos de ano do confinamento, grupo genético, periodo e ano $x$ periodo e o efeito aleatório de animal dentro de grupo genético dentro de ano. A relação MS do concentrado:MS da silagem foi incluida como covariável no modelo. Posteriormente, as características foram analisadas por um modelo de regressão que incluiu coeficientes representando as frações esperadas de Ab nos genótipos dos animais e das mães e as heterozigoses individual e materna. As médias para CMS, CA e GMD foram 2,44kg de MS/100kg de PV/dia, $6,97 \mathrm{~kg}$ de $M S / \mathrm{kg}$ de GMD e 1,435kg/dia, respectivamente. O grupo genético influenciou o CMS $(P<0,01)$ e o GMD $(P<0,06)$. O Ab igualou-se ao AbCn apresentando maior $C M S$ e menor GMD que o Cn e o CnAb. Não houve heterose para qualquer das características indicando que o cruzamento alternado Cn x Ab seria igual à média das raças paternas.

Palavras-chave: bovinos de corte, consumo de matéria seca, conversão alimentar, ganho de peso.

\section{SUMMARY}

Daily dry matter intake (CMS), feed conversion (CA) and average daily gain (ADG) of 118 Canchim (Cn), Aberdeen Angus $(\mathrm{Ab})$ and reciprocal crossbred (CnAb and $\mathrm{AbCn}$ ) males were analyzed. The CnAb group included $F_{1}, 3 / 4 C n+1 / 4 A b, 5 / 8 C n+3 / 8 A b$ and $11 / 16 C n+5 / 16 A b$. The AbCn included $F_{1}, 5 / 8 A b+3 / 8 C n$ and $11 / 16 \mathrm{Ab}+5 / 16 \mathrm{Cn}$. These animals were fed in individual stalls for 84 to 95 days receiving corn silage ad libitum plus a concentrate containing $17.8 \% \mathrm{CP}$ and $79 \%$ TDN fed $1 \%$ of live animal weight per day. The traits were first analyzed by a model that included the fixed effects of year, genetic group, period and year $x$ period interaction and the random effect of animal within genetic group within year. The ratio between the dry matter of the concentrate to the dry matter of the silage was included as a continuous variable in the model. Later, the traits were analyzed by a multiple regression model that included coefficients for the expected fractions of $A b$ in the genotypes of animals and dams and for expected individual and maternal heterozygosities. Means for CMS, CA and ADG were, respectively, $2.44 \mathrm{~kg}$ of $D M / 100 \mathrm{~kg}$ of live weight/day, $6.97 \mathrm{~kg}$ of $D M / \mathrm{kg}$ of $A D G$ and $1.435 \mathrm{~kg} /$ day. Genetic group influenced CMS $(P<0.01)$ and $A D G(P<0.06)$. The Ab was equal to $A b C n$, showing higher CMS and lower ADG than the other two groups. There was no heterosis for any of the traits indicating that rotational crossing between Canchim and Aberdeen Angus was equal to the average of the parental breeds.

Key words: beef cattle, dry matter intake, feed efficiency, average daily gain.

\section{INTRODUÇÃO}

A terminação de bovinos de corte em confinamento é uma prática que vem crescendo no Brasil (VALADARES FILHO, 1995). Associado a esse crescimento, é cada vez maior o interesse dos pecuaristas por tecnologias que concorram para aumentar a eficiência da utilização de alimentos pelos animais confinados. Nesse contexto, uma das opções é a exploração das diferenças genéticas aditivas, entre e

\footnotetext{
${ }^{1}$ Engenheiro Agrônomo, PhD., Pesquisador do IAPAR, CP 2031, 80011-979, Curitiba, PR. E-mail: dperotto@pr.gov.br. Autor para correspondência.

${ }^{2}$ Zootecista, MSc., Pesquisador do IAPAR, CP 129, 84001-970, Ponta Grossa, PR. E-mail: moletta@pr.gov.br.

${ }^{3}$ Médico Veterinário, MSc., Pesquisador do IAPAR, CP 129, 84001-970, Ponta Grossa, PR. E-mail: iaparefm@pr.gov.br. 
dentro das raças, para características como capacidade de ingestão de alimentos (WARWICK \& COBB, 1975; OLIVEIRA et al., 1994) e taxa de conversão do alimento em peso vivo ou em componentes da carcaça (ARS-USDA, 1974). Outra possibilidade é a exploração da heterose para ganho de peso em confinamento (RESTLE \& ALVES FILHO, 1992; RESTLE \& VAZ, 1999; PEROTTO et al., 2000a).

O presente estudo teve como objetivo medir o consumo voluntário de alimentos, a conversão alimentar e o ganho de peso médio diário de bovinos machos inteiros das raças Canchim, Aberdeen Angus e de seus cruzamentos recíprocos, gerados pelo cruzamento alternado entre as duas raças, quando confinados em bases constantes de idade.

\section{MATERIAL E MÉTODOS}

Foram analisados o consumo diário de matéria seca (MS) por $100 \mathrm{~kg}$ de peso vivo (CMS), a conversão alimentar $((\mathrm{kg}$ de $\mathrm{MS}$ ingerida $/ \mathrm{kg}$ de peso ganho)/dia) (CA) e o ganho de peso médio diário em confinamento (kg/dia) (GMD) de 118 machos bovinos inteiros, sendo 22 do grupo Canchim (Cn), 40 mestiços Canchim x Aberdeen Angus (CnAb), 31 mestiços Aberdeen Angus x Canchim (AbCn) e 25 Aberdeen Angus (Ab), nascidos na Estação Experimental Fazenda Modelo, do IAPAR, em Ponta Grossa-PR, nos anos de 1994/98. O grupo Canchim x Aberdeen Angus incluía $8 \mathrm{~F}_{1}, 23 / 4 \mathrm{Cn}+1 / 4 \mathrm{Ab}, 16$ $5 / 8 \mathrm{Cn}+3 / 8 \mathrm{Ab}$ e $1411 / 16 \mathrm{Cn}+5 / 16 \mathrm{Ab}$. Por outro lado, o grupo Aberdeen Angus x Canchim incluía $8 \mathrm{~F}_{1}, 17$ $5 / 8 \mathrm{Ab}+3 / 8 \mathrm{Cn}$ e $611 / 16 \mathrm{Ab}+5 / 16 \mathrm{Cn}$.

Detalhes sobre o plano de acasalamentos que gerou esses animais e sobre o manejo dos mesmos até a desmama são descritos por PEROTTO et al. (2000b). No período após a desmama, feita sempre no início do outono, os animais foram mantidos em pastagens de Hemarthria altissima mais $1 \mathrm{~kg}$ de concentrado ( $14,7 \%$ de PB e $73 \%$ de NDT) por animal por dia durante o primeiro inverno (maio a agosto).

Antes do início do segundo inverno após a desmama, com peso médio de $325 \mathrm{~kg}$ e idade média de 658 dias, os animais foram submetidos a um período de adaptação ao confinamento de 14 dias, e em seguida confinados em baias individuais por períodos de 84 a 95 dias, quando receberam silagem de milho à vontade mais concentrado (milho em grão moído, 73\%; farelo de soja, 25\%; e mistura mineral completa, $2 \%$ ) fornecido à base de $1 \%$ do peso vivo (PV) do animal por dia, ajustado a cada pesagem realizada a intervalos de 28 dias, quando os animais eram submetidos previamente a jejum de sólidos por um período de 16 horas. Além da silagem e do concentrado, os animais receberam água à vontade. Diariamente, eram computadas as quantidades fornecidas e as sobras de silagem e de concentrado. A composição bromatológica do concentrado foi a seguinte: $88,0 \%$ de $\mathrm{MS}, 17,8 \%$ de $\mathrm{PB}, 0,45 \%$ de $\mathrm{Ca}$ e $0,37 \%$ de $\mathrm{P}$. Os teores de MS e PB e o $\mathrm{pH}$ da silagem variaram com os anos de $24,3 \%$ a $33,5 \%, 7,30 \%$ a $9,48 \%$ e 3,3 a 3,5, respectivamente. Os valores médios para a MS, $\mathrm{PB}$ e $\mathrm{pH}$ da silagem foram, respectivamente, $30,3 \%, 8,2 \%$ e 3,4 .

Os dados das três características foram analisados pelo método dos quadrados mínimos (SAS, 1994), ajustando-se o seguinte modelo linear:

$Y_{\mathrm{ijkl}}=\mu+b_{1} R+A_{i}+G_{j}+a_{k}(A x G)_{i j}+P_{l}+P x A_{i l}+\varepsilon_{i j k l}$ em que $Y_{i j k l}$ é valor observado da variável (CMS, CA, GMD) no l-ésimo período (repetição), para o késimo animal, dentro da combinação entre o i-ésimo ano e o j-ésimo grupo genético, $\mu$ representa o efeito fixo da média geral da variável (CMS, CA, GMD), $b_{1}$ é o coeficiente de regressão parcial do valor da variável (CMS, CA, GMD) sobre a proporção de MS do concentrado em relação à MS da silagem, $R$ representa a proporção de MS do concentrado em relação à MS da silagem, calculada pela fórmula: consumo de concentrado x 0,88 /consumo de silagem x $0,303, A_{i}$ é o efeito fixo do i-ésimo ano de realização do confinamento, $G_{j}$ é o efeito fixo do jésimo grupo genético, $a_{k}(A x G)_{i j}$ é o efeito aleatório do k-ésimo animal, dentro da combinação entre o iésimo ano e o j-ésimo grupo genético, $P_{l}$ é o efeito fixo do 1-ésimo período de confinamento, $P x A_{l i}$ é o efeito da interação entre período e ano e $\varepsilon_{i j k l}$ é o efeito do erro experimental.

Nessas análises, foram considerados quatro níveis do fator grupo genético ( $\mathrm{Cn}, \mathrm{CnAb}, \mathrm{AbCn}$ e $\mathrm{Ab})$, cinco níveis do fator ano $(96,97,98,99$ e 00) e três níveis do fator período (ou repetição), sendo a duração de cada período igual a 28 dias, exceto para o terceiro período de 1996, que teve duração de 39 dias. Os grupos $\mathrm{CnAb}$ e $\mathrm{AbCn}$ não foram desdobrados em classes correspondentes às composições raciais porque tal procedimento resultaria em poucas observações por subclasse. O peso ao início do confinamento ou o peso médio durante o confinamento não foram mantidos nas análises definitivas por constatar-se, em análises preliminares, que essas co-variáveis influenciavam a magnitude do efeito de grupo genético. A interação entre período e grupo genético também foi investigada em análises preliminares, mas, como não mostrou efeito $(\mathrm{P}>0,05)$ sobre qualquer das três características, foi excluída do modelo estatístico. A inclusão da proporção de MS do concentrado em relação à MS da silagem, como variável contínua no modelo estatístico, teve a finalidade de ajustar os dados para o valor médio dessa variável já que a mesma variou, em 
relação ao peso vivo do animal, em função da maior ou menor ingestão de silagem, no caso fornecida à vontade.

Posteriormente, as três características foram analisadas através de um modelo de regressão múltipla em que o efeito fixo de grupo genético foi substituído por coeficientes representando as frações esperadas de genes da raça Aberdeen Angus e as frações esperadas de locos heterozigotos, com respeito à raça de origem dos genes, nos genótipos dos animais e das respectivas mães. Essas análises foram conduzidas para se estimar a heterose e o desempenho de diferentes estratégias de uso das raças Canchim e Aberdeen Angus quanto às características sob estudo. As estimativas desses parâmetros foram computadas como funções lineares dos efeitos incluídos no modelo de regressão e testadas estatisticamente pelo teste $\mathrm{t}$.

\section{RESULTADOS E DISCUSSÃO}

Os resumos das análises de variância das três características analisadas pelo modelo que incluiu o efeito fixo de grupo genético são apresentados na tabela 1 . Os valores do $\mathrm{R}^{2}$ indicam que o modelo proposto descreveu adequadamente as diferenças entre animais para CMS, CA e GMD, apesar dos altos coeficientes de variação das duas últimas características (28,78\% e 20,66\%, respectivamente). A relação entre a MS do concentrado e a MS da silagem, cuja média foi 0,567 , mostrou efeito significativo sobre as três variáveis. Visto que o concentrado foi fornecido em função do PV, animais que ingeriram

Tabela 1 - Resumos das análises de variância do consumo diário de MS por 100kg de peso vivo, da conversão alimentar e do ganho de peso médio diário de machos bovinos Canchim, Aberdeen Angus e cruzamentos recíprocos em Ponta Grossa-PR.

\begin{tabular}{lcccc}
\hline Fontede variação ${ }^{1}$ Item & Graus de liberdade & \multicolumn{3}{c}{ Característica ${ }^{2} /$ Quadrados médios } \\
\hline & & CMS & CA & GMD \\
\cline { 2 - 5 } RCV & 1 & $1,3746^{* * *}$ & $17,51^{*}$ & $0,9777^{* * *}$ \\
Ano & 4 & $0,2693^{* * *}$ & $36,19^{* * *}$ & $0,8190^{* * *}$ \\
Grupo & 3 & $0,0860^{* *}$ & $4,70^{\mathrm{NS}}$ & $0,1645^{\mathrm{NS}}$ \\
Animal (Grupo x Ano) & 129 & $0,0205^{* * *}$ & $3,66^{\mathrm{NS}}$ & $0,0889^{\mathrm{NS}}$ \\
Período & 2 & $0,4454^{* * *}$ & $36,42^{* * *}$ & $1,5090^{* * *}$ \\
Ano*Período & 8 & $0,2577^{* * *}$ & $40,87^{* * *}$ & $1,4188^{* * *}$ \\
$\mathrm{R}^{2}$ & & 0,92 & 0,50 & 0,58 \\
C.V. (\%) & & 3,35 & 28,78 & 20,66 \\
Média & & 2,44 & 6,97 & 1,435 \\
\hline
\end{tabular}

${ }^{1} \mathrm{RCV}=$ Proporção de MS do concentrado em relação à MS da silagem, $\mathrm{R}^{2}=$ Coeficiente de determinação e C.V. $=$ Coeficiente de variação. ${ }^{2} \mathrm{CMS}=$ Consumo diário de $\mathrm{MS}$ por $100 \mathrm{~kg}$ de peso vivo animal $(\mathrm{kg}$ de $\mathrm{MS} / 100 \mathrm{~kg} / \mathrm{dia}), \mathrm{CA}=$ Conversão alimentar (kg de MS/kg de GMD) e GMD = Ganho médio diário em confinamento (kg/dia). $* * *=\mathrm{P}<0,001, * *=\mathrm{P}<0,01, *=\mathrm{P}<0,05$ e NS $=\mathrm{P}>0,05$. menores quantidades de MS da silagem, em relação à MS do concentrado, ingeriram menos MS total, por unidade de PV, e apresentaram menores GMDs. A CA aumentou e, por conseguinte, a eficiência diminuiu, com o aumento da relação MS do concentrado:MS da silagem. Uma análise complementar indicou que os grupos genéticos diferiram quanto ao consumo de MS proveniente da silagem. As médias por quadrados mínimos foram $1,49 \pm 0,013,1,59 \pm 0,010,1,56 \pm$ 0,01 , e $1,67 \pm 0,012 \mathrm{~kg}$ de MS da silagem $/ 100 \mathrm{~kg}$ de $\mathrm{PV}$, respectivamente para os grupos $\mathrm{Cn}, \mathrm{CnAb}, \mathrm{AbCn}$ e $\mathrm{Ab}$, havendo diferença estatística $(\mathrm{P}<0,05)$ para qualquer contraste entre pares de médias. Este comportamento pode explicar a pequena diferença entre aos grupos quanto ao GMD, a ser discutida mais adiante, pois o grupo com menor potencial genético para ganho de peso, o Aberdeen Angus, apresentou o maior CMS.

Ano de realização do confinamento influenciou $(\mathrm{P}<0,001)$ as três variáveis, refletindo diferenças na qualidade da silagem através dos anos. Os piores desempenhos em termos de CA e GMD corresponderam aos anos em que a silagem apresentou teores de $\mathrm{PB}$ mais baixos. $\mathrm{O}$ grupo genético mostrou efeito significativo $(\mathrm{P}<0,05)$ sobre o consumo e aproximou-se do nível de significância de $5 \%(\mathrm{P}<0,6)$ para o ganho de peso, mas não teve efeito $(\mathrm{P}>0,05)$ sobre a conversão alimentar. Animal dentro de grupo genético dentro de ano teve efeito $(\mathrm{P}<0,001)$ apenas sobre CMS. Os efeitos de período e da interação entre ano e período influenciaram fortemente as três variáveis analisadas. Examinando-se as médias das três características para os vários níveis da interação ano x período, observou-se que o alto CMS no terceiro período pode ser atribuído preponderantemente ao ano de 1996 enquanto os altos ganhos no terceiro período podem ser atribuídos aos anos de 1996 e 1999. Além disso, o fenômeno conhecido como crescimento compensatório pode ser responsável pelos altos ganhos e consumo no primeiro período.

A tabela 2 apresenta as médias por quadrados mínimos, das três características, segundo o grupo genético, o período e o ano. A média para CMS foi $2,44 \mathrm{~kg}$. Na literatura brasileira são encontrados, para esta variável, valores de $2,22 \mathrm{~kg}$ de $\mathrm{MS} / 100 \mathrm{~kg}$ de PV para machos inteiros Charolês $\mathrm{x}$ Caracu (PEROTTO et al., 2000a) a $2,71 \mathrm{~kg}$ de MS/100kg de PV para machos Charolês (CASACCIA et al., 1993). Essas diferenças podem ocorrer em função do grupo genético e do nível de concentrado na MS da dieta 
Tabela 2 - Médias por quadrados mínimos para o consumo diário de MS por 100kg de peso vivo, para a conversão alimentar e para o ganho de peso médio diário de machos bovinos segundo o grupo genético, o ano e o período experimental.

\begin{tabular}{lccc}
\hline \multirow{2}{*}{$\begin{array}{l}\text { Grupo genético }{ }^{1} / \mathrm{ano} / \\
\text { período }^{2}\end{array}$} & \multicolumn{3}{c}{ Característica $^{3}$} \\
\cline { 2 - 4 } & & CMS & GMD \\
\hline Grupo genético & & & \\
& & & \\
Canchim (Cn) & $2,41 \pm 0,011^{\mathrm{a}}$ & $6,89 \pm 0,27^{\mathrm{a}}$ & $1,487 \pm 0,040^{\mathrm{a}}$ \\
CnAb & $2,43 \pm 0,007^{\mathrm{a}}$ & $7,05 \pm 0,18^{\mathrm{a}}$ & $1,467 \pm 0,027^{\mathrm{a}}$ \\
AbCn & $2,46 \pm 0,009^{\mathrm{b}}$ & $6,54 \pm 0,22^{\mathrm{a}}$ & $1,453 \pm 0,033^{\mathrm{ab}}$ \\
Aberdeen Angus (Ab) & $2,48 \pm 0,010^{\mathrm{b}}$ & $7,05 \pm 0,24^{\mathrm{a}}$ & $1,375 \pm 0,035^{\mathrm{b}}$ \\
Ano & & & \\
1996 & $2,54 \pm 0,016^{\mathrm{a}}$ & $6,61 \pm 0,39^{\mathrm{bc}}$ & $1,503 \pm 0,058^{\mathrm{ab}}$ \\
1997 & $2,44 \pm 0,010^{\mathrm{c}}$ & $6,42 \pm 0,26^{\mathrm{c}}$ & $1,460 \pm 0,036^{\mathrm{ab}}$ \\
1998 & $2,41 \pm 0,009^{\mathrm{d}}$ & $7,32 \pm 0,22^{\mathrm{ab}}$ & $1,305 \pm 0,032^{\mathrm{c}}$ \\
1999 & $2,34 \pm 0,011^{\mathrm{e}}$ & $6,36 \pm 0,27^{\mathrm{c}}$ & $1,561 \pm 0,040^{\mathrm{a}}$ \\
2000 & $2,48 \pm 0,009^{\mathrm{b}}$ & $7,71 \pm 0,23^{\mathrm{a}}$ & $1,399 \pm 0,034^{\mathrm{b}}$ \\
Período & & & \\
I & & & \\
II & $2,49 \pm 0,009^{\mathrm{a}}$ & $6,43 \pm 0,23^{\mathrm{b}}$ & $1,470 \pm 0,034$ \\
III & $2,38 \pm 0,008^{\mathrm{b}}$ & $7,48 \pm 0,20^{\mathrm{a}}$ & $1,294 \pm 0,030$ \\
& $2,46 \pm 0,014^{\mathrm{a}}$ & $6,73 \pm 0,34^{\mathrm{ab}}$ & $1,573 \pm 0,050$ \\
\hline
\end{tabular}

${ }^{1} \mathrm{CnAb}=$ Mestiços Canchim $\mathrm{x}$ Aberdeen Angus, filhos de touros Canchim, $\mathrm{AbCn}$ = Mestiço Aberdeen Angus x Canchim, filhos de touros Aberdeen Angus.

${ }^{2}$ Cada período teve a duração de 28 dias, exceto o terceiro período de 1996 que durou 39 dias.

${ }^{3} \mathrm{CMS}=$ Consumo diário de MS por $100 \mathrm{~kg}$ de peso vivo animal $(\mathrm{kg}$ de $\mathrm{MS} / 100 \mathrm{~kg} / \mathrm{dia}), \mathrm{CA}=$ Conversão alimentar $(\mathrm{kg}$ de $\mathrm{MS} / \mathrm{kg}$ de GMD) e GMD = Ganho médio diário em confinamento ( $\mathrm{kg} / \mathrm{dia})$.

Para a mesma fonte de variação, médias na mesma coluna, sobrescritas pela mesma letra, não diferem $(\mathrm{P}>0,05)$ pelo teste $\mathrm{t}$.

ingerida pelos animais. Os resultados da tabela 2 indicam que animais Canchim e mestiços filhos de touros Canchim apresentaram menores valores $(\mathrm{P}<0,05)$ de CMS que os Aberdeen Angus e os mestiços filhos de touros Aberdeen Angus. MOLETTA \& RESTLE (1992) encontraram maior consumo de MS/100kg de PV em novilhos Angus comparados a novilhos Charolês e Nelore. RESTLE $\boldsymbol{e t}$ al. (1995) trabalhando com Charolês, Nelore e seus $F_{1}$ recíprocos e PEROTTO et al. (2000a) estudando Charolês, Caracu e cruzamentos recíprocos de gerações avançadas do esquema alternado de cruzamentos Charolês x Caracu observaram pequenas diferenças para consumo de MS por $100 \mathrm{~kg}$ de $\mathrm{PV}$ em função do grupo genético. Por outro lado, EUCLIDES FILHO et al. (1997) não encontraram diferenças para esta característica entre machos inteiros dos grupos Nelore e cruzamentos de Nelore com Charolês, Fleckvieh e Chianina. RESTLE et al. (2000) também não detectaram diferenças significativas para $o$ CMS/100kg de PV entre grupos de novilhos de diferentes genótipos Hereford x Nelore. Os números de animais por grupo genético no presente trabalho são superiores aos informados nos trabalhos citados. Isto resulta em menores erros-padrão e, assim, em maiores probabilidades de detecção de diferenças entre grupos.

As médias para CA variaram de $6,54 \pm 0,22 \mathrm{~kg}$ de $\mathrm{MS} / \mathrm{kg}$ de GMD para o grupo $\mathrm{AbCn}$ a $7,05 \pm 0,24 \mathrm{~kg}$ de $\mathrm{MS} / \mathrm{kg}$ de GMD para o $\mathrm{CnAb}$, sem diferenças entre grupos. EUCLIDES FILHO et al. (1997) também não encontraram diferenças quanto à conversão alimentar entre animais Nelore e cruzamentos 3/4 Nelore $+1 / 4$ Bos taurus (Charolês, Fleckvieh e Chianina). Geralmente, esta característica não é influenciada pelo grupo genético quando os animais são levados até o mesmo grau de acabamento, exceto se o nível de concentrado na dieta for alto, caso em que as raças mais precoces são mais eficientes (RESTLE $\boldsymbol{e t}$ al., 1983; LANNA, 1997). PEROTTO et al. (2000a) encontraram diferenças significativas entre Charolês e Caracu bem como entre os mestiços recíprocos dessas duas raças para a conversão alimentar, mas destacaram que as comparações foram feitas com animais da mesma faixa etária, caso em que os animais menos precoces são mais eficientes (LANNA, 1997).

O GMD variou de $1,375 \mathrm{~kg} / \mathrm{d}$ para o Aberdeen Angus a $1,487 \mathrm{~kg} / \mathrm{d}$ para o Canchim, sendo significativa $(\mathrm{P}<0,05)$ a diferença entre esse dois grupos, fato que está de acordo com as diferenças aditivas entre as duas raças para velocidade de crescimento e peso adulto. Diferenças entre grupos raciais, com diferentes potenciais genéticos para peso adulto, comparados em bases constantes de peso ou de idade, quanto ao GMD em confinamento, são comuns na literatura (EUCLIDES FILHO $\boldsymbol{e}$ t al., 1997; PEROTTO $\boldsymbol{e}$ t al., 2000a; RESTLE et al., 2000). A falta de diferença estatística para o GMD em confinamento entre os grupos Angus e Charolês, reportada por MOLETTA \& RESTLE (1992) pode ser atribuída à deficiência da dieta oferecida aos animais ( $72 \%$ de cana-de-açúcar + $28 \%$ de concentrado em base de MS).

A tabela 3 apresenta resultados obtidos pelas análises em que o grupo genético foi substituído por coeficientes de regressão parcial representando os efeitos genéticos direto e materno do Aberdeen Angus, expressos como desvios em relação aos mesmos efeitos do Canchim, e os efeitos heterozigóticos direto e materno. Este procedimento oferece a possibilidade de se estimar o desempenho de grupos genéticos que não estiveram presentes no experimento e de estimar a heterose como coeficiente de regressão parcial do desempenho sobre a fração esperada de locos 
Tabela 3 - Estimativas de efeitos genéticos e do desempenho de diferentes estratégias de uso das raças Canchim e Aberdeen Angus quanto ao consumo de MS por $100 \mathrm{~kg}$ de peso vivo, à conversão alimentar e ao ganho de peso médio diário em Ponta Grossa-PR.

\begin{tabular}{lccc}
\hline Efeito $^{1} /$ Estratégia $^{2}$ & \multicolumn{3}{c}{ Característica $^{3}$} \\
\cline { 2 - 4 } & & CMS & GMD \\
\hline Efeito & $0,008 \pm 0,017$ & $0,136 \pm 0,304$ & $0,062 \pm 0,050$ \\
Heterose do $F_{1}$ & $0,004 \pm 0,024$ & $0,013 \pm 0,212$ & $0,033 \pm 0,035$ \\
Heterose retida & & & \\
Estratégia & $0,034 \pm 0,024$ & $0,391 \pm 0,428$ & $0,023 \pm 0,070$ \\
$(1 / 2 \mathrm{Cn}+1 / 2 \mathrm{Ab})-\mathrm{Cn}$ & $0,035 \pm 0,018^{*}$ & $-0,176 \pm 0,318$ & $0,021 \pm 0,052$ \\
$(1 / 2 \mathrm{Ab}+1 / 2 \mathrm{Cn})-\mathrm{Cn}$ & $-0,018 \pm 0,022$ & $0,448 \pm 0,397$ & $0,102 \pm 0,065$ \\
$(1 / 2 \mathrm{Cn}+1 / 2 \mathrm{Ab})-\mathrm{Ab}$ & $-0,017 \pm 0,0021^{*}$ & $-0,119 \pm 0,379$ & $0,101 \pm 0,062$ \\
$(1 / 2 \mathrm{Ab}+1 / 2 \mathrm{Cn})-\mathrm{Ab}$ & $0,031 \pm 0,015$ & $-0,015 \pm 0,281$ & $-0,007 \pm 0,046$ \\
Cruzamento Alternado $-\mathrm{Cn}$ & $-0,021 \pm 0,013$ & $0,042 \pm 0,234$ & $0,073 \pm 0,038^{*}$ \\
Cruzamento Alternado $-\mathrm{Ab}$ & & & \\
\hline
\end{tabular}

${ }^{1}$ Heterose do $\mathrm{F}_{1}=\frac{\left(\frac{1}{2} C n+\frac{1}{2} A b\right)+\left(\frac{1}{2} A b+\frac{1}{2} C n\right)}{2}-\frac{(C n+A b)}{2}$

Heterose retida $=\frac{\left(\frac{63}{100} C n+\frac{37}{100} A b\right)+\left(\frac{63}{100} A b+\frac{37}{100} C n\right)}{2}-\frac{(C n+A b)}{2}$,

${ }^{2} \mathrm{Cn}=$ Canchim e $\mathrm{Ab}=$ Aberdeen Angus

${ }^{3} \mathrm{CMS}=$ Consumo diário de MS por $100 \mathrm{~kg}$ de peso vivo animal $(\mathrm{kg}$ de $\mathrm{MS} / 100 \mathrm{~kg} / \mathrm{dia}), \mathrm{CA}=$ Conversão alimentar ( $\mathrm{kg}$ de MS/kg de GMD) e GMD = Ganho médio diário em confinamento ( $\mathrm{kg} / \mathrm{dia})$.

$*=\mathrm{P}<0,05$ pelo teste $\mathrm{t}$.

heterozigotos nos genótipos dos animais. Não houve heterose para qualquer das três características analisadas nem na primeira geração de cruzamentos tampouco nas gerações avançadas do cruzamento alternado Canchim x Aberdeen Angus, como indicam as estimativas da heterose do $F_{1}$ e da heterose retida. Geralmente, as respostas heteróticas dependem do nível esperado de heterozigoze, com respeito às raças de origem dos genes, nas populações em estudo, da divergência genética entre as raças envolvidas nos cruzamentos e das magnitudes das herdabilidades das características consideradas.

Embora não significativas, as estimativas da heterose retida são menores que as do $F_{1}$, o que está de acordo com o menor nível de heterozigose esperado nas gerações avançadas do cruzamento alternado em relação à primeira geração. As três características aqui estudadas têm altas herdabilidades. Os valores médios das estimativas relacionadas por PRESTON \& WILLIS (1974) são 0,48 para CMS, 0,46 para CA e 0,52 para GMD. Desse modo, os valores das heteroses apresentados na tabela 3 condizem com as expectativas teóricas determinadas pela importância dos efeitos aditivos na expressão das características.

PEROTTO et al. (2000a) não encontraram heterose significativa para CMS em cruzamentos envolvendo gerações avançadas do esquema alternado Charolês $\mathrm{x}$ Caracu. Em trabalhos envolvendo as raças Charolês, Nelore e seus $F_{1}$ recíprocos (RESTLE \& ALVES FILHO, 1992; CASACCIA et al., 1993; RESTLE et al., 1995), as estimativas de heterose para o consumo de $\mathrm{MS} / 100 \mathrm{~kg}$ de PV variaram de $-9,2 \%$ a $+3,9 \%$. Em outro trabalho, RESTLE et al. (2000) não detectaram diferença significativa para consumo de MS/100kg de PV entre animais Hereford $\mathrm{x}$ Nelore de diferentes composições raciais, o que sugere ausência de heterose para a característica. Os resultados desses autores concordam com os do presente trabalho, confirmando que a heterose para consumo de MS em relação ao peso vivo é próxima de zero.

As estimativas de heterose para a conversão alimentar encontradas na literatura variam de $6 \% \mathrm{a}+7 \%$ (PRESTON \& WILLIS, 1974). LONG (1980), em ampla revisão sobre o assunto, concluiu que a heterose para esta característica é próxima a $1 \%$. Assim, a falta de significância estatística para a estimativa desse parâmetro sobre a CA no presente trabalho, bem como no de PEROTTO et al. (2000a), está de acordo com a expectativa teórica baseada na herdabilidade e com os resultados da literatura.

Para GMD, PEROTTO et al. (2000a) encontraram heterose da ordem de $7 \%$ em cruzamentos Charolês x Caracu. Os resultados de RESTLE \& ALVES FILHO (1992) e de RESTLE \& VAZ (1999) apresentam estimativas de heterose, para a mesma característica, de 9,3\% e de 13,3\%, na geração $F_{1}$ Nelore $x$ Charolês. Para retro-cruzados Charolês x Nelore, RESTLE \& VAZ (1999) encontraram heterose de $11,4 \%$. Nesses casos, as divergências genéticas entre Charolês e Caracu e entre Nelore e Charolês, maiores que a existente entre Angus e Canhim, podem explicar as heteroses mais altas. Os resultados também estão de acordo com a revisão de PRESTON \& WILLIS (1974), que afirmam, com base em mais de quarenta trabalhos, ser o ganho de peso até o abate, a característica pósdesmama que melhor responde à heterose.

Os contrastes $(1 / 2 \mathrm{Cn}+1 / 2 \mathrm{Ab})-\mathrm{Cn},(1 / 2 \mathrm{Ab}$ $+1 / 2 \mathrm{Cn})-\mathrm{Cn},(1 / 2 \mathrm{Cn}+1 / 2 \mathrm{Ab})-\mathrm{Ab}$ e $(1 / 2 \mathrm{Ab}+$ $1 / 2 \mathrm{Cn})$ - Ab comparam os $\mathrm{F}_{1}$ com cada uma das raças 
paternas para CA nem para GMD. Assim, pode-se afirmar que, à exceção do CMS, os esquemas de cruzamentos específicos (produção exclusiva de $\mathrm{F}_{1}$ ) entre Canchim e Angus são iguais a qualquer das duas raças paternas quanto ao desempenho em confinamento. Os resultados da tabela 3 mostram ainda que a implementação de um esquema alternado de cruzamentos entre $\mathrm{Cn}$ e $\mathrm{Ab}$ teria, para as três características, o mesmo desempenho do Canchim, mas seria superior $(\mathrm{P}<0,05)$ ao $\mathrm{Ab}$ para GMD.

\section{CONCLUSÕES}

Quando comparados na mesma faixa etária, animais das raças Canchim e Aberdeen Angus apresentam os mesmos desempenhos quanto à conversão alimentar. No entanto, diferem quanto ao consumo de matéria seca por unidade de peso vivo e ao ganho de peso médio diário em confinamento.

Mestiços resultantes dos cruzamentos entre Canchim e Aberdeen Angus não apresentam heterose para as características de desempenho em confinamento; entretanto, os animais das gerações avançadas do cruzamento alternado entre Canchim e Aberdeen Angus produzem mestiços com maior velocidade de ganho de peso que a dos animais Aberdeen Angus.

\section{REFERÊNCIAS BIBLIOGRÁFICAS}

ARS - (AGRICULTURAL RESEARCH SERVICE - USDA). Germplasm evaluation program progress report. Clay Center, Nebraska-USA : U.S. Meat Animal Research Center, 1974. ARS-NC-13. (Report № 1).

CASACCIA, J.L, PIRES, C.C, RESTLE, J. Confinamento de bovinos inteiros ou castrados de diferentes grupos genéticos. In: REUNIÃO ANUAL DA SOCIEDADE BRASILEIRA DE ZOOTECNIA, 30, 1993, Rio de Janeiro. Anais... Rio de Janeiro : SBZ, 1993. p.468.

EUCLIDES FILHO, K., EUCLIDES, V.P.B., FIGUEIREDO, G.R., et al. Avaliação de animais Nelore e seus mestiços com Charolês, Fleckvieh e Chianina, em três dietas. 1. Ganho de peso e Conversão alimentar. Revista Brasileira de Zootecnia, v.26, n.1, p.66-72, 1997.

LANNA, D.P. Fatores condicionantes e predisponentes da puberdade e da idade de abate. In: SIMPÓSIO SOBRE PECUÁRIA DE CORTE, 4, 1996. Piracicaba, SP. Anais... Piracicaba : FEALQ, 1997. p.41-78.

LONG, C.R. Crossbreeding for beef production: experimental results. Journal of Animal Science, v.51, n.5, p.1197-1123, 1980.
MOLETTA, J.L., RESTLE, J. Desempenho em confinamento de novilhos de diferentes grupos genéticos. Ciência Rural, v.22, n.2, p.227-233, 1992.

OLIVEIRA, M.A.T., FONTES, C.A.A., LANA, R.P., $\boldsymbol{e} \boldsymbol{t} \boldsymbol{a l}$. Consumo alimentar e digestibilidade de rações com dois níveis de concentrado em bovinos de cinco grupos genéticos. Revista da Sociedade Brasileira Zootecnia, v.23, n.4, p.667-677, 1994.

PEROTTO, D., MOLETTA, J.L., OLIVEIRA, J.E.P., et al. Consumo e conversão alimentar de machos bovinos inteiros Charolês, Caracu e cruzamentos recíprocos em confinamento. Revista Brasileira de Zootecnia, v.29, n.1, p.108-116. 2000a.

PEROTTO, D., CUBAS, A.C., MOLETTA, J.L., et al. Heterose sobre os pesos de bovinos Canchim e Aberdeen Angus e de seus cruzamentos recíprocos. Pesquisa Agropecuária Brasileira, v.35, n.12, p.2511-2520, 2000b.

PRESTON, T.R., WILLIS, M.B. Intensive Beef Production. 2.ed. Oxford: Pergamon, 1974. 567p.

RESTLE, J., EUCLIDES FILHO, K. HARGROVE, D.D., et al. Feedlot performance of Angus, Brown Swiss, F1, F2 and backcross steers. Journal of Animal Science, v.57, Supplement 1, p.25. 1983.

RESTLE, J., ALVES FILHO, D.C. Confinamento de terneiros inteiros ou castrados de diferentes grupos genéticos. In: REUNIÃO ANUAL DA SOCIEDADE BRASILEIRA DE ZOOTECNIA, 29, 1992. Lavras, MG. Anais... Lavras : SBZ, 1992. p. 186

RESTLE, J., VAZ, F.N. Confinamento de bovinos definidos e cruzados. In: LOBATTO, J.F.P., BARCELLOS, J.O.J., KESSLER, A.M. Produção de bovinos de corte. Porto Alegre: EDIPUCRS, 1999. p.141-167.

RESTLE, J., FELTEN, H.G., VAZ, F.N. Efeito da raça e heterose para desempenho em confinamento de novilhos de corte. In: REUNIÓN LATINO-AMERICANA DE PRODUCCIÓN ANIMAL, 14, 1995, Mar del Plata. Memorias... Balcarce: ALPA, 1995. p.852-854

RESTLE, J., QUADROS, A.R.B., VAZ, F.N. Terminação em confinamento de novilhos de diferentes genótipos Hereford $\mathrm{x}$ Nelore. Revista Brasileira de Zootecnia, v.29, n.1, p.125-130. 2000.

SAS Institute Inc. SAS/STAT User's guide. Version 6, Fourth Edition. Cary, NC : SAS Institute Incorporatin, 1994. Volume 2. 1686p.

VALADARES FILHO, S.C. Nutrição de bovinos de corte: problemas e perspectivas. In: REUNIÃO ANUAL DA SOCIEDADE BRASILEIRA DE ZOOTECNIA, 32, 1995. Brasília, DF. Anais... Brasília : SBZ, 1995. p.156-162.

WARWICK, E.J., COBB, E.H. Genetic variation in nutrition of cattle for meat production. In: THE EFFECT OF GENETIC VARIANCE ON NUTRITIONAL REQUIREMENTS OF ANIMALS, 1974, College Park, Maryland, USA. Proceedings... Washington, D.C. : National Academy of Sciences, 1975. p.3-18.

Ciência Rural, v. 32, n. 4, 2002. 